\title{
Long non-coding RNA CASC15 facilitates esophageal squamous cell carcinoma tumorigenesis via decreasing SIM2 stability via FTO-mediated demethylation
}

\author{
BO QIN ${ }^{1}$, MENG DONG $^{2}$, ZHENGYANG WANG $^{3}$, JIAJIA WAN ${ }^{4}$, YINGYING XIE ${ }^{1}$, YI JIAO $^{5}$ and DAN YAN ${ }^{1}$ \\ ${ }^{1}$ Translational Medical Center, Departments of ${ }^{2}$ Oncology and ${ }^{3}$ Pathology; ${ }^{4}$ Medical Research Center, \\ The First Affiliated Hospital of Zhengzhou University, Zhengzhou, Henan 450052; \\ ${ }^{5}$ Yongcheng Coal \& Electricity Holding Group Co., Ltd., Shangqiu, Henan 476000, P.R. China
}

Received July 7, 2020; Accepted October 27, 2020

DOI: 10.3892/or.2020.7917

\begin{abstract}
Long non-coding RNAs (lncRNAs) are involved in the regulation of esophageal squamous cell carcinoma (ESCC) progression. However, the function and mechanism of lncRNA cancer susceptibility candidate 15 (CASC15) are poorly defined. In the present study, tumor and normal adjacent tissues were collected from 45 patients with ESCC. Expression levels of CASC15, fat mass and obesity-associated (FTO) protein and single-minded 2 (SIM2) were examined via reverse transcription-quantitative PCR and western blot assays. Cell proliferation and apoptosis were evaluated via MTT, flow cytometry and caspase-3 activity assays, respectively. Additionally, an ESCC mouse xenograft model was used to assess the function of CASC15 in vivo. The interaction between FTO and CASC15/SIM2 was analyzed via RNA immunoprecipitation and RNA pull-down assays. The results revealed that CASC15 expression was elevated in ESCC tissues, and patients with ESCC exhibiting high CASC15 expression had a poor prognosis. CASC15-knockdown inhibited ESCC cell proliferation and facilitated apoptosis. Additionally, CASC15-knockdown decreased the growth of ESCC xenograft tumors. CASC15 decreased SIM2 stability via FTO-mediated demethylation. Additionally, FTO loss markedly weakened CASC15-mediated pro-proliferative and anti-apoptotic effects in ESCC cells. SIM2 downregulation weakened the effect of CASC15-knockdown on cell proliferation and inhibited the increase of the apoptotic rate and caspase-3 activity induced by CASC15 depletion in ESCC cells. In conclusion, CASC15
\end{abstract}

Correspondence to: Dr Bo Qin, Translational Medical Center, The First Affiliated Hospital of Zhengzhou University, 1 Eastern Jianshe Road, Erqi, Zhengzhou, Henan 450052, P.R. China

E-mail: fccqinb@zzu.edu.cn

Key words: esophageal squamous cell carcinoma, cancer susceptibility candidate 2, fat mass and obesity-associated protein, single-minded 2 promoted ESCC tumorigenesis by decreasing SIM2 stability via FTO-mediated demethylation.

\section{Introduction}

Esophageal cancer is a fatal tumor that affects numerous individuals worldwide (1). It was estimated that $\sim 572,034$ new cases and 508,585 deaths of esophageal cancer occurred globally in 2018, accounting for $3.2 \%$ of all cancer diagnoses and $5.3 \%$ of all cancer-associated deaths (1). Esophageal squamous cell carcinoma (ESCC) accounts for $~ 90 \%$ of all cases of esophageal cancer (2). The main risk factors of ESCC are tobacco smoking and excess alcohol consumption (3). Despite substantial improvements in elucidating the progression and improving the treatment of ESCC, the outcome of patients remains unsatisfactory (4). Hence, novel strategies for the treatment of ESCC are required.

Long non-coding RNAs (lncRNAs), a class of non-coding RNAs ( $>200$ nucleotides in length), have been found to be involved in the progression and treatment resistance of ESCC $(5,6)$. For instance, IncRNA cancer susceptibility candidate 2 (CASC2) inhibits ESCC cell proliferation, migration and invasion by modulating suppressor of cytokine signaling 1 (7). Additionally, IncRNA LOC100133669 facilitates ESCC cell proliferation by regulating Tim50 (8). CASC15 is an oncogenic factor that has been identified in several types of cancer, such as tongue squamous cell carcinoma, oral squamous cell carcinoma, melanoma and cervical cancer (9-12). Furthermore, CASC15 expression is upregulated in ESCC (13). However, little is known about the function and mechanism of CASC15 in ESCC progression.

N6-methyladenosine (m6A) modifications serve a vital role in cancer progression by controlling mRNA metabolism, including pre-mRNA splicing, mRNA transport, translation and stability $(14,15)$. For instance, the m6A RNA demethylase fat mass and obesity-associated (FTO) regulates acute myeloid leukemia cell viability and differentiation by targeting the 3'-untranslated regions of ankyrin repeat and SOCS box containing 2 and retinoic acid receptor $\alpha$ transcripts (16). Methyltransferase-like 3 promotes gastric cancer progression by enhancing heparin binding growth factor mRNA stability 
via m6A modification (17). Previous studies have demonstrated that lncRNAs regulate cell fates by affecting mRNA expression via m6A RNA modification (18-20). CASC15 is predicted to interact with the m6A methylation-related protein FTO according to the StarBase database (http://starbase.sysu.edu. $\mathrm{cn} / \mathrm{rbpClipRNA}$.php? source $=$ lncRNA \& flag $=$ RBP \&clade $=\mathrm{ma}$ mmal\&genome $=$ human \&assembly $=$ hg $19 \& R B P=F T O \& c l i p N$ $\mathrm{um}=\&$ panNum=\&target $=$ ). FTO is highly expressed in patients with ESCC and FTO dysregulation is closely associated with the progression of ESCC (21). Bioinformatics analysis and previous RNA-seq data (13) reveal that single-minded 2 (SIM2) expression is markedly downregulated and negatively associated with FTO expression in ESCC. Moreover, previous studies have demonstrated that SIM2 exerts vital roles in ESCC progression (22-24). However, whether SIM2 is required for CASC15-mediated ESCC progression remains uncertain. Considering that FTO may interact with SIM2 and CASC15, the present study hypothesized that CASC15 may be involved in the regulation of ESCC progression by FTO-meditated SIM2 mRNA demethylation.

In the current study, the expression level of CASC15 in ESCC tumors and its roles in ESCC progression were investigated. Furthermore, whether CASC15 exerted its functions by regulating SIM2 via FTO in ESCC was assessed.

\section{Materials and methods}

Patients and tissue collection. Paired tumor and normal adjacent tissues ( $>5 \mathrm{~cm}$ from the edge of tumor tissues) were harvested from 45 patients (age range, 43-80 years; mean age, 65 years) with ESCC admitted to the First Affiliated Hospital of Zhengzhou University (Zhengzhou, China) and the Hospital of Yongcheng Coal \& Electricity Holding Group Co., Ltd. (Shangqiu, China) between September 2013 and December 2015. None of the patients had received any treatment prior to surgery. The clinical features of patients are presented in Table I. Tumors were staged using the 7 th edition of the American Joint Committee on Cancer Tumor-Node-Metastasis staging system for $\operatorname{ESCC}(25,26)$. The overall survival of patients was analyzed after a 5-year follow-up. Written informed consent was obtained from all patients. The present study was approved by the Ethics Committee of the First Affiliated Hospital of Zhengzhou University and conducted in accordance with the Declaration of Helsinki. Tumor and adjacent normal tissue samples were stored at $-80^{\circ} \mathrm{C}$.

Cell culture. Human ESCC cell lines (Eca109 and KYSE450 cells) and an esophageal epithelial cell line (HET-1A) were provided by Fenghui Biotech. HET-1A cells were cultured in serum-free Bronchial Epithelial Cell Growth medium containing bovine pituitary extract, insulin, hydrocortisone, GA-1000, retinoic acid, transferrin, triiodothyronine, epinephrine and human epidermal growth factor (all Procell Life Science \& Technology Co., Ltd.). Eca109 and KYSE450 cells were cultured in RPMI-1640 medium (Procell Life Science \& Technology Co., Ltd.) supplemented with $10 \%$ FBS (HyClone; Cytiva) and $1 \%$ penicillin/streptomycin (Sangon Biotech Co., Ltd.) in a $5 \% \mathrm{CO}_{2}$ incubator at $37^{\circ} \mathrm{C}$.
Cell transfection. FTO and CASC15 overexpression vectors were constructed using the pcDNA3.1 vector (Thermo Fisher Scientific, Inc.). The pcDNA3.1 vector alone was used as a negative control (pcDNA). Small interfering RNAs (siRNAs/si) for CASC15 (si-CASC15\#1, 5'-AAUUCAUGA UGCUUUCUAGAU-3'; si-CASC15\#2, 5'-AGAACUUAA CCCAAAAGUCCA-3'; si-CASC15\#3, 5'-AUACAGUUC CUUAUAUGAGGG-3'), SIM2 (si-SIM2, 5'-ACAUUUCAU UCGAAGAAAGAA-3') and the scrambled negative control (si-NC; 5'-AAGACAUUGUGUGUCCGCCTT-3') were generated by Guangzhou RiboBio Co., Ltd. Eca109 and KYSE450 cells were transfected with $800 \mathrm{ng}$ vector or $20 \mathrm{nM}$ siRNAs using Lipofectamine ${ }^{\circledR} 3000$ reagent (Thermo Fisher Scientific, Inc.). Transfection was performed at room temperature and the complexes of transfection reagent and DNA/siRNA were co-incubated for $15 \mathrm{~min}$. Transfection efficacy was tested $24 \mathrm{~h}$ post-transfection.

Reverse transcription-quantitative PCR (RT-qPCR). Total RNA derived from tissues and cells was isolated using TRIzol ${ }^{\circledR}$ (Vazyme Biotech Co., Ltd.) as previously described (27). RNA (800 ng) was reverse transcribed into cDNA using the M-MLV reverse transcription kit (Thermo Fisher Scientific, Inc.) according to the manufacturer's protocol. cDNA was mixed with SYBR Green (Toyobo Life Science) and specific primers (GenScript). The mixture was subsequently used for RT-qPCR with the following thermocycling conditions: $95^{\circ} \mathrm{C}$ for $5 \mathrm{~min}$, followed by 40 cycles of $95^{\circ} \mathrm{C}$ for $20 \mathrm{sec}$ and $60^{\circ} \mathrm{C}$ for $1 \mathrm{~min}$. The primer sequences were as follows: CASC15 forward, 5'-CTT TGTCTGCTCCGGGACTT-3' and reverse, 5'-TTAAGGGAC ATTTCCCCCGC-3'; SIM2 forward, 5'-CTAGCCACACAT CGCGGG-3' and reverse, 5'-CTCGGCTCCGGGCATATT AG-3'; $\beta$-actin forward, 5'-CTTCGCGGGCGACGAT-3' and reverse, 5'-CCACATAGGAATCCTTCTGACC-3'. Using $\beta$-actin as a reference, relative mRNA expression was calculated using the $2^{-\Delta \Delta \mathrm{Cq}}$ method (28).

MTT assay. Cell proliferation was examined by performing an MTT assay. Eca109 and KYSE450 cells were seeded into 96-well plates at a density of $1 \times 10^{4}$ cells/well and cultured for $0,24,48$ or $72 \mathrm{~h}$ at $37^{\circ} \mathrm{C}$. MTT $(15 \mu \mathrm{l}$; Beyotime Institute of Biotechnology) at a final concentration of $0.5 \mathrm{mg} / \mathrm{ml}$ was added to each well. After incubation for $4 \mathrm{~h}$ at $37^{\circ} \mathrm{C}$, medium was replaced with $100 \mu \mathrm{l}$ DMSO (Beijing Solarbio Science \& Technology Co., Ltd.). Optical density values were subsequently detected at $570 \mathrm{~nm}$ using a microplate reader (Beijing Potenov Technology Co. Ltd.).

Flow cytometry. An Annexin V-APC/7-AAD apoptosis kit (Beijing Biolab Technology Co., Ltd.) was used to measure cell apoptosis via flow cytometry. Eca109 and KYSE450 cells were seeded in 12-well plates at a density of $1 \times 10^{5}$ cells/well. After culture for $72 \mathrm{~h}$ at $37^{\circ} \mathrm{C}$, cells were harvested and re-suspended in Annexin $\mathrm{V}$ binding buffer. Cells were then stained with Annexin V-APCand 7-AAD for 15 min at room temperature in the dark, and examined using a flow cytometer (NovoCyte 2060R; Agilent Technologies, Inc.) and NovoExpress ${ }^{\mathrm{TM}}$ software v1.0 (Agilent Technologies, Inc.). Apoptotic rate was determined from the ratio of $\mathrm{APC}^{+} / 7-\mathrm{AAD}^{-}$(early apoptotic cells) and $\mathrm{APC}^{+} / 7-\mathrm{AAD}^{+}$cells (late apoptotic cells). 
Table I. Association between CASC15 expression and clinicopathological features of patients with esophageal squamous cell carcinoma.

Relative CASC15 expression

\begin{tabular}{|c|c|c|c|}
\hline \multirow[b]{2}{*}{ Clinicopathological features } & & \multirow[b]{2}{*}{ P-value } \\
\hline & High, n (\%) & Low, n (\%) & \\
\hline Sex & & & 0.6677 \\
\hline Male & $14(53.8)$ & $12(46.2)$ & \\
\hline Female & $9(47.4)$ & $10(52.6)$ & \\
\hline Age, years & & & 0.6718 \\
\hline$\geq 65$ & $15(53.6)$ & $13(46.4)$ & \\
\hline$<65$ & $8(47.1)$ & $9(52.9)$ & \\
\hline Tumor size, $\mathrm{cm}$ & & & 0.2078 \\
\hline$\geq 5$ & $10(66.7)$ & $5(33.3)$ & \\
\hline$<5$ & $13(43.3)$ & $17(56.7)$ & \\
\hline TNM stage & & & 0.0045 \\
\hline $\mathrm{I}+\mathrm{II}$ & $10(34.5)$ & $19(65.5)$ & \\
\hline III & $13(72.2)$ & $3(37.8)$ & \\
\hline Lymphatic metastasis & & & 0.0006 \\
\hline No & 11(34.4) & $21(65.6)$ & \\
\hline Yes & $12(92.3)$ & $1(7.7)$ & \\
\hline
\end{tabular}

Caspase-3 activity assay. Caspase-3 activity was measured using a caspase-3 Assay kit (Abcam) in accordance with the manufacturer's protocol. Briefly, Eca109 and KYSE450 cells were cultured for $72 \mathrm{~h}$ at $37^{\circ} \mathrm{C}$. Next, $1 \times 10^{6}$ cells were digested with trypsin and washed 3 times with PBS. Cell pellets were collected through low-speed centrifugation at $800 \mathrm{x} g$ for $5 \mathrm{~min}$ at room temperature. Next, cells were re-suspended in chilled cell lysis buffer and incubated for $10 \mathrm{~min}$ on ice. After centrifugation $\left(10,000 \mathrm{x} \mathrm{g}\right.$ for $1 \mathrm{~min}$ at $\left.4^{\circ} \mathrm{C}\right)$, cell supernatants were transferred to a fresh tube and protein concentration was determined using a bicinchoninic acid assay kit (Beyotime Institute of Biotechnology). Subsequently, samples were incubated with DEVD-p-NA substrate (final concentration, $200 \mu \mathrm{M}$ ) for $2 \mathrm{~h}$ at $37^{\circ} \mathrm{C}$. Finally, absorbance at $400 \mathrm{~nm}$ was examined using a microplate reader and relative caspase-3 activity was normalized to that of the control group.

Western blotting. Cells were lysed using RIPA buffer (Beijing Solarbio Science \& Technology Co., Ltd.) supplemented with $1 \%$ protease inhibitor (Thermo Fisher Scientific, Inc.). After high-speed centrifugation $\left(12,000 \mathrm{x}\right.$ g for $15 \mathrm{~min}$ at $\left.4^{\circ} \mathrm{C}\right)$, cell supernatants were collected and total protein was quantified using a bicinchoninic acid assay kit (Beyotime Institute of Biotechnology). Protein samples (20 $\mu \mathrm{g} /$ lane) were separated via $10 \%$ SDS-PAGE and transferred onto nitrocellulose membranes (Beijing Solarbio Science \& Technology Co., Ltd.). After blocking with 5\% bovine serum albumin (Sigma-Aldrich; Merck $\mathrm{KGaA}$ ) for $1 \mathrm{~h}$ at room temperature, membranes were incubated overnight at $4^{\circ} \mathrm{C}$ with the following primary antibodies (all from Abcam): FTO (cat. no. ab126605; 1:10,000), SIM2 (cat. no. ab131161; 1:1,000) and $\beta$-actin (cat. no. ab8227; 1:3,000). Samples were then incubated at room temperature with horseradish peroxidase-conjugated secondary antibodies (cat. no. ab205718; 1:10,000; Abcam) for $2 \mathrm{~h}$. The resultant blots were exposed to ECL reagent (Abcam) and analyzed using ImageJ v1.8.0 software (National Institutes of Health).

Bioinformatics analysis. The interaction between CASC15 and FTO was predicted using the StarBase v2.0 database (The Encyclopedia of RNA Interactomes; http://starbase.sysu.edu. $\mathrm{cn} / \mathrm{rbpClipRNA}$.php? source $=\operatorname{lncRNA} \&$ flag $=$ target $\&$ clade $=\mathrm{m}$ ammal\&genome $=$ human $\&$ assembly $=$ hg $19 \&$ RBP $=$ all\&clipN $\mathrm{um}=1 \&$ panNum=0\&target $=$ CASC15). The mRNAs that may interact with the FTO protein were searched using StarBase v2.0 database (http://starbase.sysu.edu.cn/rbpClipRNA.php?so urce $=$ mRNA\&flag $=$ RBP $\&$ clade $=$ mammal $\&$ genome $=$ human $\&$ assembly=hg19\&RBP=FTO\&clipNum=\&panNum=\&target=). The correlation analysis between FTO and other genes in ESCC was performed using StarBase v2.0 database (http://starbase. sysu.edu.cn/panGeneCoExp.php ). The expression pattern of CASC15 and SIM2 in esophagus cancer and normal tissues was predicted using the Gene Expression Profiling Interactive Analysis (GEPIA) database (http://gepia.cancer-pku.cn/detail. php?gene $=\&$ clicktag=boxplot $)$.

Mouse xenograft experiments. 293T cells (The Cell Bank of Type Culture Collection of the Chinese Academy of Sciences) were maintained in DMEM medium (Thermo Fisher Scientific, Inc.) containing 10\% FBS (HyClone; Cytiva) in a $5 \% \mathrm{CO}_{2}$ incubator at $37^{\circ} \mathrm{C}$. A total of $18 \mathrm{BALB} / \mathrm{c}$ athymic mice (male; 5-week-old; $\sim 16 \mathrm{~g}$ ) was purchased from Charles River Laboratories, Inc. Mice were raised at $20-25^{\circ} \mathrm{C}$ in a specific pathogen-free environment with clean air, $60 \%$ relative humidity and a 12-h dark/light cycle, with free access to water and food. A short hairpin (sh)RNA fragment was subcloned into the pLKO.1 vector (Addgene, Inc.) and 
sh-NC or sh-CASC15 lentiviruses were generated by Hanbio Biotechnology Co., Ltd. Briefly, $1 \mu \mathrm{g}$ pLKO.1 shRNA plasmid was transfected into 293T cells with 750 ng of psPAX2 packaging (Addgene, Inc.) and $250 \mathrm{ng}$ pMD2.G envelope plasmids using Lipofectamine ${ }^{\circledR} 3000$ reagent (Thermo Fisher Scientific, Inc.). At 36 and $60 \mathrm{~h}$ after transfection, media containing lentiviral particles were harvested. Next, cells in the media were removed using a $0.45-\mu \mathrm{m}$ filter and lentiviral particles were collected. KYSE450 cells were transduced with sh-CASC15 or sh-NC lentiviruses (multiplicity of infection=5) for $24 \mathrm{~h}$. After $48 \mathrm{~h}$ of lentivirus infection, cells were screened using puromycin $(3 \mu \mathrm{g} / \mathrm{ml})$ for 14 days to establish stably transduced cell lines. Mice of the sh-CASC15 or sh-NC group ( $n=6$ per group) were subcutaneously injected with KYSE450 cells $\left(1 \times 10^{6}\right.$ per mouse) stably transfected with sh-CASC15 or sh-NC, respectively. Mice $(n=6)$ of the sham group were injected with $2 \times 10^{6}$ KYSE450 cells without transfection. Cells were resuspended in PBS solution. Tumor volume was examined every 7 days and calculated using the following formula: Length $\mathrm{x}$ width ${ }^{2} / 2$ (29). On day 35 post-injection, mice were anesthetized using 5\% isoflurane and then euthanized by the intraperitoneal injection of sodium pentobarbital $(150 \mathrm{mg} / \mathrm{kg})$. Tumors were resected, imaged and weighed. CASC15 expression in tumor tissues was measured via RT-qPCR. The protocol was approved by the Institutional Animal Care and Use Committee of the First Affiliated Hospital of Zhengzhou University.

RNA pull-down assay. RNA pull-down analysis was conducted using the Magnetic RNA-Protein Pull-Down kit (cat. no. 20164; Thermo Fisher Scientific, Inc.) in accordance with the manufacturer's protocol. Briefly, cells were lysed using Pierce IP Lysis Buffer (cat. no. 87787; Thermo Fisher Scientific, Inc.) for $10 \mathrm{~min}$ and then centrifuged at $13,000 \mathrm{x}$ g for $10 \mathrm{~min}$ at $4^{\circ} \mathrm{C}$ to pellet the cell debris and obtain cell lysates (cell supernatants). Cell lysates ( $25 \mu \mathrm{l}$ per IP reaction) were co-incubated with nucleic acid-compatible streptavidin magnetic beads (Pierce; Thermo Fisher Scientific, Inc.) combined with biotinylated sense or antisense CASC15 (Sangon Biotech Co., Ltd.). Proteins of the RNA-protein complexes were eluted from the magnetic beads by boiling $\left(8 \mathrm{~min}\right.$ at $\left.100^{\circ} \mathrm{C}\right)$, and FTO protein expression was examined as aforementioned via western blotting. The antibody against FTO (cat. no. ab126605; 1:10,000) used for western blotting was purchased from Abcam, as aforementioned.

RNA immunoprecipitation (RIP) assay. RIP analysis was performed using the Magna RIP RNA-Binding Protein Immunoprecipitation kit (cat. no. 17-701; EMD Millipore) along with antibodies against IgG (cat. no. 12-370/12-371; 1:100; Sigma-Aldrich; Merck KGaA), FTO (cat. no. ABE552; 1:100; Sigma-Aldrich; Merck KGaA) or m6A (cat. no. MABE1006; 1:100; Sigma-Aldrich; Merck KGaA). Cells were collected by centrifugation at $800 \mathrm{x}$ g for $5 \mathrm{~min}$ at $4^{\circ} \mathrm{C}$ and then lysed using RIP lysis buffer (EMD Millipore) supplemented with protease and RNase inhibitors (EMD Millipore). Cell lysates were incubated for $5 \mathrm{~min}$ on ice and stored at $-80^{\circ} \mathrm{C}$. Antibody was incubated for $1 \mathrm{~h}$ at room temperature with magnetic beads Protein A/G. Next, the compounds of antibody and magnetic beads in $900 \mu \mathrm{l}$ RIP Immunoprecipitation Buffer were co-incubated overnight at $4^{\circ} \mathrm{C}$ with cell lysates supernatants $(100 \mu \mathrm{l})$ after high-speed centrifugation $(13,000 \mathrm{x} \mathrm{g}$ for
$10 \mathrm{~min}$ at $4^{\circ} \mathrm{C}$ ). Subsequently, the beads were washed 6 times using cold RIP Wash Buffer. RNA on the beads was eluted, extracted and purified following the instructions of the manufacturer. CASC15 and SIM2 mRNA expression was detected via RT-qPCR, as aforementioned.

mRNA stability analysis. To detect the stability of SIM2, transfected Eca109 and KYSE450 cells were incubated at $37^{\circ} \mathrm{C}$ with $2 \mu \mathrm{g} / \mathrm{ml}$ Actinomycin D (ActD; Amyjet Scientific, Inc.) for 1, 2, 3 or $4 \mathrm{~h}$. RNA was subsequently extracted, and SIM2 mRNA expression was determined via RT-qPCR, as aforementioned.

Statistical analysis. Statistical analysis was performed using GraphPad Prism 6 (GraphPad Software, Inc.). Data were presented as the mean $\pm \mathrm{SD}$ of triplicate experiments. The overall survival curve was generated and assessed via Kaplan-Meier and log-rank tests. Associations between CASC15 expression and clinicopathological parameters of patients with ESCC were analyzed using the $\chi^{2}$ test (for analyzing sex and age) or Fisher's exact test (for analyzing tumor size, lymphatic metastasis and TNM stage) in Table I. The linear correlation was analyzed using Pearson's correlation analysis and two-tailed t-test. Paired t-tests were used in the comparisons between tumor and adjacent normal tissues. Unpaired t-tests were used in the comparisons between two groups of cells. Differences among $\geq 3$ groups were analyzed using one-way ANOVA followed by Tukey's post-hoc test, standard two-way ANOVA followed by Bonferroni's post-hoc test or mixed two-way ANOVA followed by Bonferroni's post-hoc test. $\mathrm{P}<0.05$ was considered to indicate a statistically significant difference.

\section{Results}

CASC15 expression is increased in ESCC tumor tissues. To determine whether CASC15 was involved in the regulation of ESCC progression, CASC15 expression in ESCC was assessed by GEPIA and RT-qPCR. GEPIA analysis revealed that CASC15 expression was significantly increased in ESCC tumor tissues compared with in normal tissues (Fig. 1A). Furthermore, the RT-qPCR results revealed that CASC15 expression was 3.5-fold higher in ESCC tissues compared with in normal samples (Fig. 1B). To analyze the association of CASC15 expression and the prognosis of patients with ESCC, individuals were divided into high $(n=23)$ or low $(n=22)$ CASC15 groups according to the median level of CASC15 expression in tumor tissues. Patients with high CASC15 expression demonstrated lower overall survival rates compared with the low CASC15 expression group $(\mathrm{P}=0.0218$; Fig. 1C). Furthermore, CASC15 expression was significantly associated with TNM stage and lymphatic metastasis, but not with tumor size, sex and age (Table I). The results indicated that CASC15 expression was increased in ESCC tumor tissues and associated with poor ESCC patient outcomes.

CASC15-knockdown decreases ESCC cell proliferation and promotes apoptosis. To determine the function of CASC15 in ESCC cells in vitro, CASC15 expression was assessed in ESCC cell lines. As presented in Fig. 2A, the expression 
A

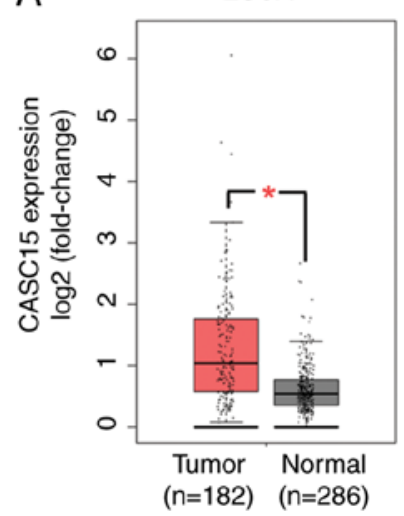

B

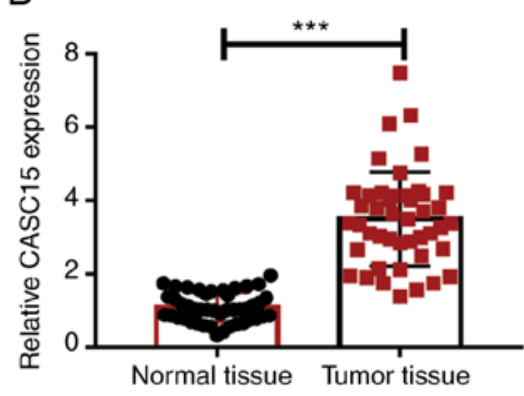

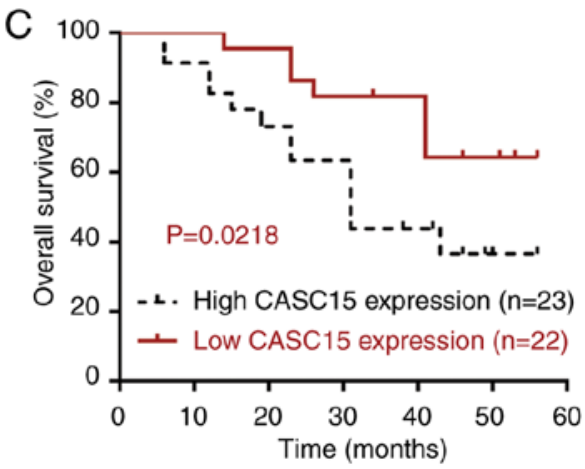

Figure 1. CASC15 expression is increased in ESCC tumor tissues. (A) Expression analysis of CASC15 expression in ESCC was conducted using the Gene Expression Profiling Interactive Analysis database. (B) CASC15 expression was detected via reverse transcription-quantitative PCR in the tumor and adjacent normal tissues of patients with ESCC $(n=45)$. (C) Overall survival of patients with ESCC was analyzed in high and low CASC15 expression groups. Data were expressed as the mean \pm SD from three experiments. ${ }^{*} \mathrm{P}<0.05$ and ${ }^{* * *} \mathrm{P}<0.001$. CASC15, cancer susceptibility candidate 15; ESCC, esophageal squamous cell carcinoma.
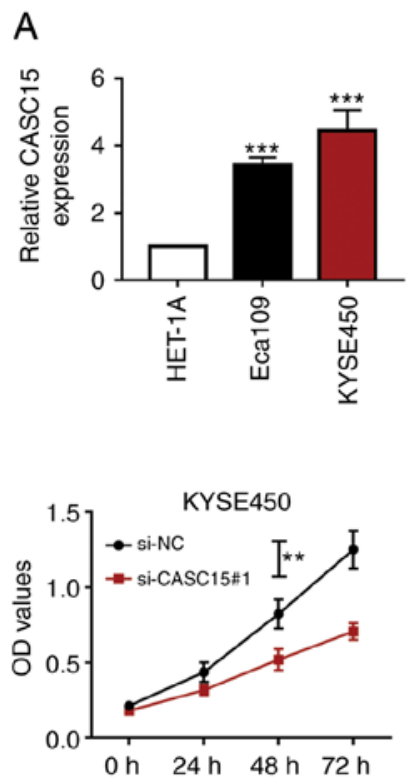

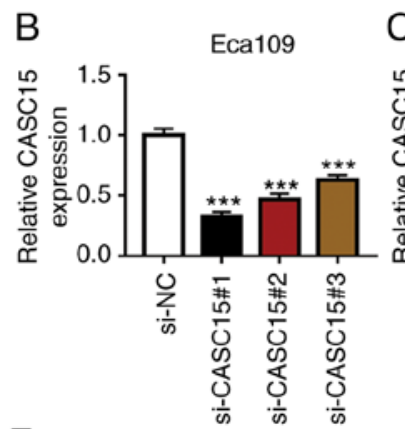

$\mathrm{E}$

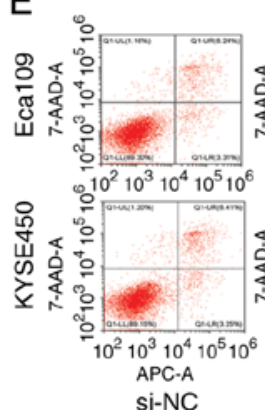

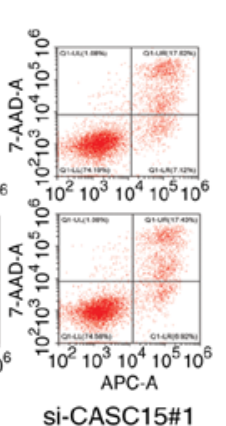
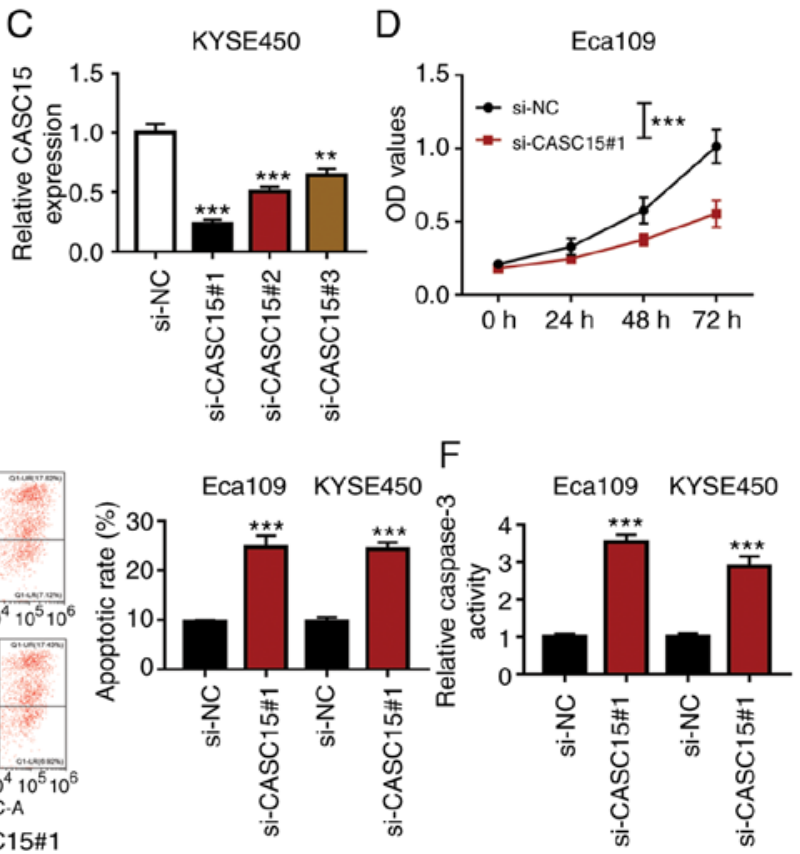

Figure 2. Effects of CASC15-knockdown on ESCC cell proliferation and apoptosis. (A) CASC15 expression was determined via RT-qPCR in ESCC (Eca109 and KYSE450) and HET-1A cell lines. CASC15 expression was assessed via RT-qPCR in (B) Eca109 and (C) KYSE450 cells transfected with si-CASC15\#1-3 or si-NC. Data analyzed via one-way ANOVA followed by Tukey's post-hoc test. (D) The proliferative ability of Eca109 and KYSE450 cells transfected with si-CASC15\#1 or si-NC was assessed via an MTT assay at 0,24, 48 and $72 \mathrm{~h}$. Data analyzed via two-way ANOVA followed by Bonferroni's post-hoc test. (E) The apoptotic rate of Eca109 and KYSE450 cells transfected with si-CASC15\#1 or si-NC was measured via flow cytometry. (F) Relative caspase-3 activity was examined in Eca109 and KYSE450 cells transfected with si-CASC15\#1 or si-NC. Data were expressed as the mean \pm SD from three experiments. ${ }^{* *} \mathrm{P}<0.01$ and ${ }^{* * *} \mathrm{P}<0.001$ vs. HET-1A or si-NC. CASC15, cancer susceptibility candidate 15 ; ESCC, esophageal squamous cell carcinoma; RT-qPCR, reverse transcription-quantitative PCR; si, small interfering RNA; NC, negative control; OD, optical density.

levels of CASC15 mRNA in Eca109 and KYSE450 cells were $>3.5$-fold higher compared with in HET-1A cells. In subsequent experiments, CASC15 expression in Eca109 and KYSE450 cells was knocked down using si-CASC15\#1, si-CASC15\#2 or si-CASC15\#3 (Fig. 2B and C). The results revealed that si-CASC15\#1 demonstrated the highest knockdown efficacy $(>70 \%)$ and was therefore used for subsequent experiments. MTT analysis revealed that CASC15-knockdown significantly inhibited the proliferation of Eca109 and KYSE450 cells (Fig. 2D). Furthermore, the flow cytometry results demonstrated that CASC15 silencing induced a 2 .6-fold increase in cell apoptotic rate (Q1-UR+Q1-LR) in Eca109 cells (Fig. 2E). Additionally, a 2.5 -fold upregulation in cell apoptotic rate (Q1-UR+Q1-LR) was observed in CASC15-depleted KYSE450 cells compared with in the si-NC control group (Fig. 2E). Additionally, CASC15 downregulation significantly increased caspase-3 activity in Eca109 and KYSE450 cells (Fig. 2F). The results indicated that CASC15 silencing repressed ESCC progression in vitro.

CASC15 silencing inhibits ESCC xenograft tumor growth in vivo. To determine the role of CASC 15 in ESCC progression 

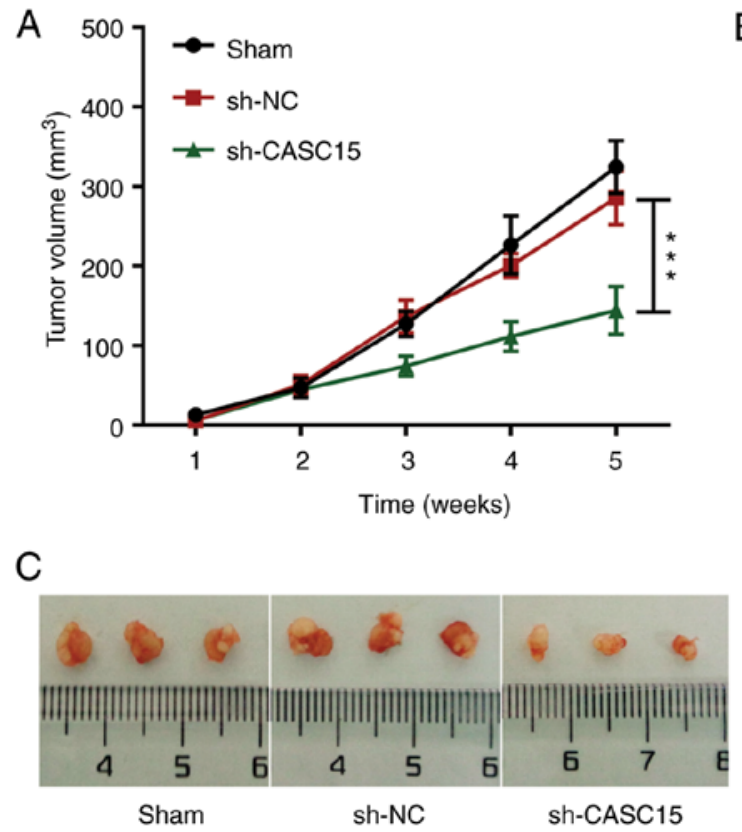

B

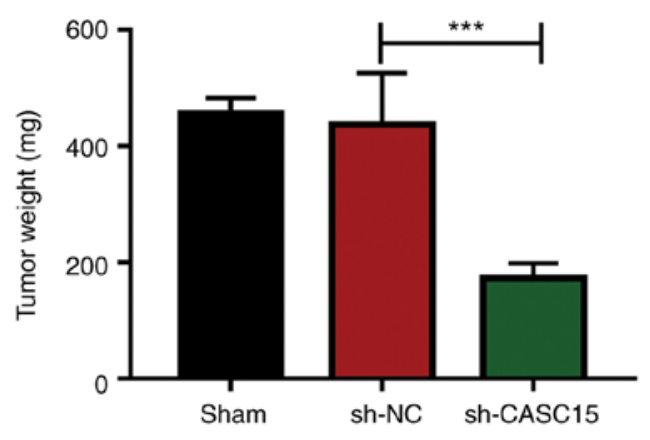

D

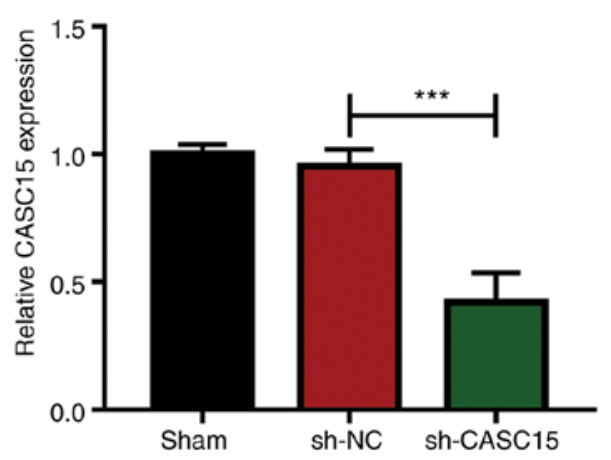

Figure 3. Effect of CASC15-knockdown on esophageal squamous cell carcinoma xenograft tumor growth. (A) Tumor volume was detected every 7 days in each group. Data analyzed via mixed two-way ANOVA followed by Bonferroni's post-hoc test. (B) Tumor weight was measured on day 35 after injection in each group. (C) Representative images of tumors. (D) CASC15 expression was detected via reverse transcription-quantitative PCR in the tumor tissues of each group. Data analyzed via one-way ANOVA followed by Tukey's post-hoc test. ${ }^{* * *} \mathrm{P}<0.001$. CASC15, cancer susceptibility candidate 15 ; sh, short hairpin; NC, negative control.

in vivo, KYSE450 cells with relatively higher levels of CASC15 compared with Eca109 cells were used to establish the ESCC mouse xenograft model. Mice were divided into sham (non-transfected group), sh-NC or sh-CASC15 groups after cells were injected following transfection. On day 35 after injection, tumor volume and weight were significantly decreased in the sh-CASC15 group compared with in the sham or sh-NC group (Fig. 3A-C). Additionally, CASC15 expression in the tumor tissues of each group was detected. The results revealed that CASC15 expression was significantly decreased in the sh-CASC15 group compared with in the sham or sh-NC group (Fig. 3D). These data indicated that CASC15 silencing hindered ESCC xenograft tumor growth.

CASC1 inhibits SIM2 expression and decreases SIM2 mRNA stability via FTO in ESCC cells. Considering the vital roles of RNA binding proteins (RBPs) in the regulation of gene expression and cancer progression, RBPs that may interact with CASC15 were predicted using the StarBase database. The results are presented in Table II. Among the identified RBPs, m6A methylation-associated FTO protein was selected for further assessment. The RNA pull-down assay revealed that FTO was substantially enriched by biotin-labeled sense CASC15 in Eca109 cells (Fig. 4A), indicating that CASC15 interacted with the FTO protein in ESCC cells. Combined with StarBase database prediction data for FTO-binding mRNAs and previous RNA-seq data (13), 19 downregulated genes (BARX2, MXD1, LNX1, AGFG2, GMDS, SESN2, VAV3, LPIN1, CYP2J2, PIM1, PAQR5, RMND5B, NAPRT, SIM2, CSTB, GPT2, CES2, YOD1 and TREX2) were identified in
ESCC (Fig. 4B). The differences in expression levels of these 19 genes in ESCC tumors compared with in normal tissues are presented in Table III.

StarBase Pearson's correlation analysis was performed to explore the correlation of the mRNA expression levels of FTO and those of the 19 downregulated genes in ESCC. Among the 19 genes, 12 genes were negatively correlated with FTO mRNA expression in ESCC (Table IV). Among these 12 genes, only SIM2 has been implicated in ESCC progression in previous studies $(22,23)$. Additionally, the GEPIA database revealed that SIM2 expression was significantly decreased in ESCC tumor tissues compared with in normal tissues (Fig. 4C). Consequently, SIM2 was selected as a potential downstream target of FTO in subsequent experiments.

Subsequent RIP and RT-qPCR assays revealed that CASC15 and SIM2 mRNA expression was significantly increased in FTO immunoprecipitation complexes compared with IgG immunoprecipitation complexes in Eca109 cells (Fig. 4D), indicating a close association between CASC15, FTO and SIM2. It is widely known that FTO exerts its functions by m6A RNA demethylation (30). In the current study, the effect of FTO overexpression on SIM2 and CASC15 m6A methylation were assessed via RIP and RT-qPCR assays. Transfection efficiency analysis revealed that the transfection of FTO overexpression plasmid led to a marked increase in FTO protein expression in Eca109 cells (Fig. 4E). The results of RIP and RT-qPCR assays revealed that FTO overexpression significantly decreased the m6A methylation level of SIM2, but did not affect the m6A methylation level of CASC15 in Eca109 cells (Fig. 4E). The CASC15 
Table II. Predicted RBPs that interact with CASC15.

\begin{tabular}{|c|c|c|c|c|c|c|}
\hline $\mathrm{RBP}$ & Gene ID & Gene name & Gene type & Cluster no. & Clip Exp no. & Clip ID no. \\
\hline ADAR & ENSG00000272168 & CASC15 & lincRNA & 89 & 3 & 89 \\
\hline CELF2 & ENSG00000272168 & CASC15 & lincRNA & 123 & 2 & 187 \\
\hline CNBP & ENSG00000272168 & CASC15 & lincRNA & 1 & 1 & 1 \\
\hline DDX54 & ENSG00000272168 & CASC15 & lincRNA & 3 & 3 & 3 \\
\hline DGCR8 & ENSG00000272168 & CASC15 & lincRNA & 17 & 4 & 25 \\
\hline DHX9 & ENSG00000272168 & CASC15 & lincRNA & 5 & 4 & 5 \\
\hline DKC1 & ENSG00000272168 & CASC15 & lincRNA & 31 & 1 & 31 \\
\hline EIF4A3 & ENSG00000272168 & CASC15 & lincRNA & 49 & 2 & 53 \\
\hline ELAVL1 & ENSG00000272168 & CASC15 & lincRNA & 46 & 4 & 50 \\
\hline EWSR1 & ENSG00000272168 & CASC15 & lincRNA & 10 & 2 & 12 \\
\hline FBL & ENSG00000272168 & CASC15 & lincRNA & 21 & 2 & 22 \\
\hline FMR1 & ENSG00000272168 & CASC15 & lincRNA & 2 & 1 & 2 \\
\hline FTO & ENSG00000272168 & CASC15 & lincRNA & 1 & 1 & 1 \\
\hline FUS & ENSG00000272168 & CASC15 & lincRNA & 119 & 3 & 121 \\
\hline HNRNPA1 & ENSG00000272168 & CASC15 & lincRNA & 1 & 2 & 2 \\
\hline HNRNPA2B1 & ENSG00000272168 & CASC15 & lincRNA & 4 & 3 & 6 \\
\hline HNRNPC & ENSG00000272168 & CASC15 & lincRNA & 6 & 3 & 6 \\
\hline HNRNPD & ENSG00000272168 & CASC15 & lincRNA & 1 & 1 & 1 \\
\hline HNRNPM & ENSG00000272168 & CASC15 & lincRNA & 139 & 4 & 139 \\
\hline IGF2BP2 & ENSG00000272168 & CASC15 & lincRNA & 131 & 4 & 141 \\
\hline LARP7 & ENSG00000272168 & CASC15 & lincRNA & 1 & 1 & 1 \\
\hline LIN28A & ENSG00000272168 & CASC15 & lincRNA & 2 & 1 & 2 \\
\hline MBNL2 & ENSG00000272168 & CASC15 & lincRNA & 4 & 1 & 4 \\
\hline METTL14 & ENSG00000272168 & CASC15 & lincRNA & 1 & 1 & 1 \\
\hline METTL3 & ENSG00000272168 & CASC15 & lincRNA & 1 & 1 & 1 \\
\hline MOV10 & ENSG00000272168 & CASC15 & lincRNA & 2 & 2 & 2 \\
\hline MSI1 & ENSG00000272168 & CASC15 & lincRNA & 42 & 3 & 42 \\
\hline NOP56 & ENSG00000272168 & CASC15 & lincRNA & 14 & 1 & 14 \\
\hline NOP58 & ENSG00000272168 & CASC15 & lincRNA & 9 & 2 & 9 \\
\hline PTBP1 & ENSG00000272168 & CASC15 & lincRNA & 19 & 2 & 21 \\
\hline RBFOX2 & ENSG00000272168 & CASC15 & lincRNA & 582 & 4 & 628 \\
\hline RBM10 & ENSG00000272168 & CASC15 & lincRNA & 1 & 1 & 1 \\
\hline RBM5 & ENSG00000272168 & CASC15 & lincRNA & 2 & 1 & 2 \\
\hline SRSF1 & ENSG00000272168 & CASC15 & lincRNA & 1 & 1 & 1 \\
\hline SRSF10 & ENSG00000272168 & CASC15 & lincRNA & 3 & 2 & 3 \\
\hline SRSF3 & ENSG00000272168 & CASC15 & lincRNA & 1 & 1 & 1 \\
\hline TAF15 & ENSG00000272168 & CASC15 & lincRNA & 94 & 5 & 100 \\
\hline TARDBP & ENSG00000272168 & CASC15 & lincRNA & 467 & 5 & 531 \\
\hline TROVE2 & ENSG00000272168 & CASC15 & lincRNA & 1 & 1 & 1 \\
\hline U2AF2 & ENSG00000272168 & CASC15 & lincRNA & 3 & 6 & 6 \\
\hline UPF1 & ENSG00000272168 & CASC15 & lincRNA & 17 & 4 & 18 \\
\hline YTHDC1 & ENSG00000272168 & CASC15 & lincRNA & 5 & 3 & 5 \\
\hline
\end{tabular}

CASC15, cancer susceptibility candidate 15; RBP, RNA-binding protein; lincRNA, long non-coding RNA; Clip Exp, crosslinking-immunoprecipitation experiment.

overexpression plasmid was subsequently constructed and si-FTO was synthesized to further explore the regulatory networks between CASC15, FTO and SIM2 in ESCC. Transfection efficiency analysis revealed that the transfection of CASC15 overexpression plasmid in Eca109 and
KYSE450 cells significantly upregulated CASC15 expression compared with the pcDNA-transfected group (Fig. 4F). Additionally, a marked downregulation of FTO protein expression was observed in si-FTO-transfected Eca109 and KYSE450 cells compared with in si-NC-transfected cells 


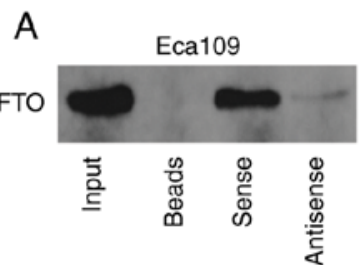

D

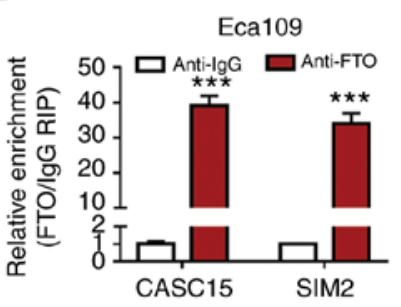

B

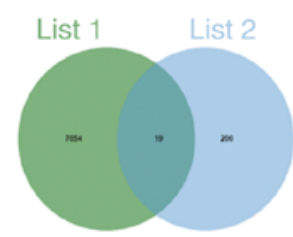

List1: Predicted genes interacting with FTO List2: Differentially expressed genes

\section{E}
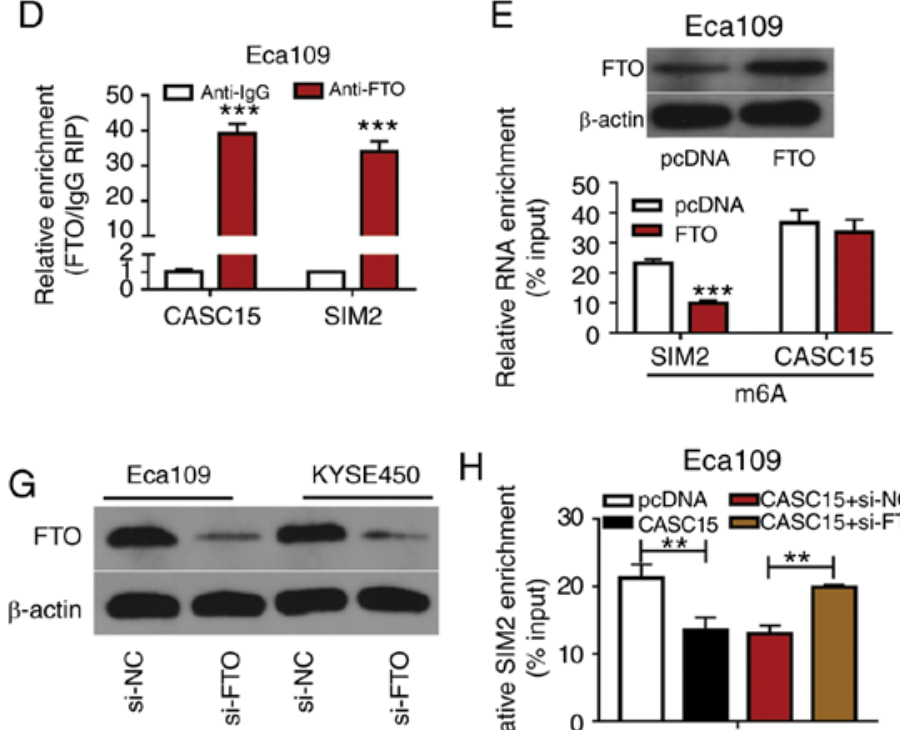

Eca109

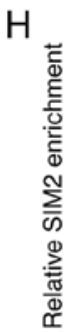

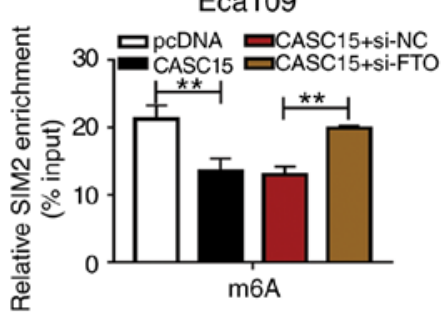

C

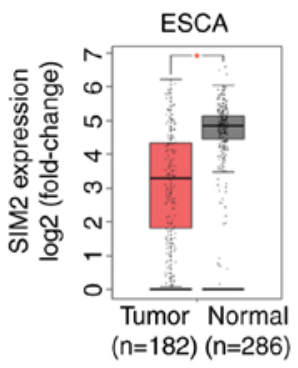

$\mathrm{F}$

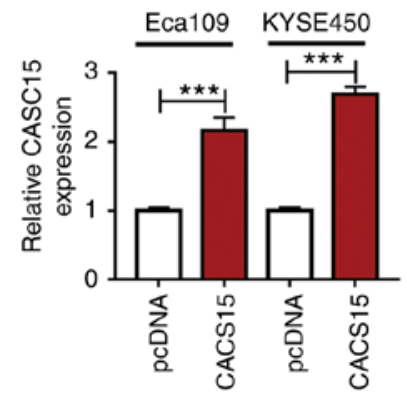

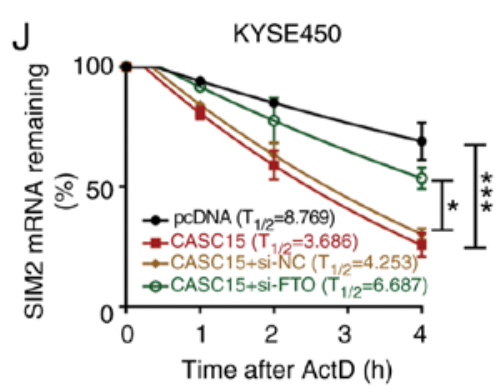

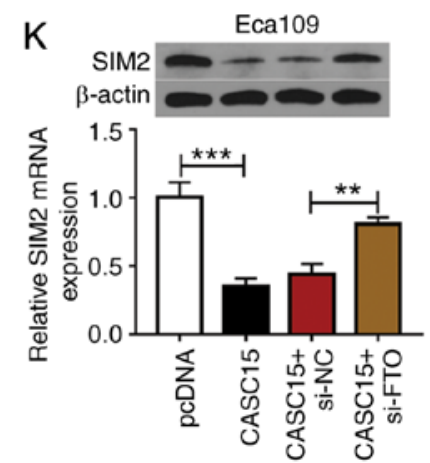

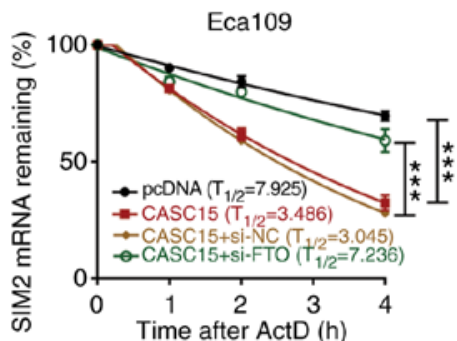

KYSE450

$\mathrm{L}$
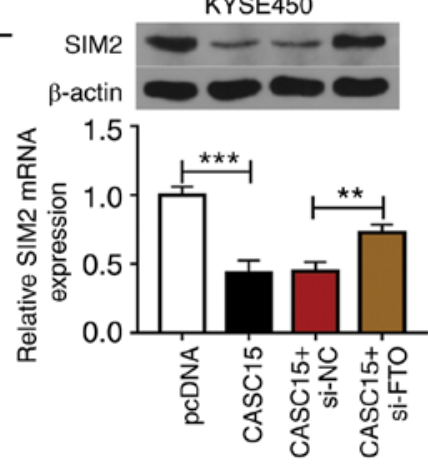

Figure 4. CASC15 inhibited SIM2 expression and decreased SIM2 mRNA stability via FTO in esophageal squamous cell carcinoma cells. (A) RNA pull-down and western blotting assays were performed to determine the interaction of CASC15 and FTO protein. (B) Venn diagram of the possible mRNAs that interact with the FTO protein, as determined using the StarBase database and previous RNA-seq data. (C) SIM2 expression was assessed in ESCC tumor and adjacent normal tissues using the Gene Expression Profiling Interactive Analysis database. (D) CASC15 and SIM2 mRNA expression enriched by IgG or FTO antibodies were detected via RT-qPCR after a RIP assay in Eca109 cells. (E) Eca109 cells were transfected with pcDNA or the FTO overexpression vector. At $48 \mathrm{~h}$ post transfection, FTO protein expression was measured via western blotting, and m6A-enriched SIM2 and CASC15 mRNA expression was examined through RIP and RT-qPCR assays. Transfection efficiency of the (F) CASC15 overexpression vector and (G) si-FTO was measured in Eca109 and KYSE450 cell lines. (H) Eca109 cells were transfected with pcDNA, CASC15 overexpression vector, CASC15 overexpression vector + si-NC or CASC15 overexpression vector + si-FTO. At $48 \mathrm{~h}$ post transfection, RIP and RT-qPCR assays were conducted to measure SIM2 mRNA expression following m6A antibody enrichment. SIM2 mRNA stability was analyzed by performing an ActD assay in (I) Eca109 and (J) KYSE450 cells transfected with pcDNA, CASC15 overexpression vector, CASC15 overexpression vector + si-NC or CASC15 overexpression vector + si-FTO. Data analyzed via two-way ANOVA followed by Bonferroni's post-hoc test. SIM2 mRNA and protein expression was respectively examined via RT-qPCR and western blotting in (K) Eca109 and (L) KYSE450 cells transfected with pcDNA, CASC15 overexpression vector, CASC15 overexpression vector + si-NC or si-FTO. ${ }^{*} \mathrm{P}<0.05,{ }^{* *} \mathrm{P}<0.01$ and ${ }^{* * * *} \mathrm{P}<0.001$. CASC15, cancer susceptibility candidate 15; SIM2, single-minded 2; FTO, fat mass and obesity-associated; RIP, RNA immunoprecipitation; RT-qPCR, reverse transcription-quantitative PCR; si, small interfering RNA; NC, negative control; m6A, n6-methyladenosine; ActD, Actinomycin D.

(Fig. 4G). CASC15 overexpression significantly decreased SIM2 mRNA m6A methylation levels in Eca109 cells, while the downregulation of FTO expression significantly weakened this effect (Fig. 4H). The current study further assessed whether CASC15 influenced SIM2 mRNA stability and expression via FTO. The results demonstrated that CASC15 overexpression significantly decreased SIM2 mRNA stability and inhibited SIM2 mRNA and protein expression in Eca-109 and KYSE450 cells (Fig. 4I-L). In addition, FTO suppression significantly enhanced SIM2 mRNA stability and expression, 
Table III. Differentially expressed genes in esophagus cancer that interact with fat mass and obesity-associated protein.

\begin{tabular}{lll}
\hline Gene name & $\log$ (fold-change) & P-value \\
\hline BARX2 & -3.40645 & $3.53 \times 10^{-7}$ \\
MXD1 & -2.34351 & $8.63 \times 10^{-8}$ \\
LNX1 & -3.02622 & $3.45 \times 10^{-9}$ \\
AGFG2 & -2.44104 & $7.9 \times 10^{-7}$ \\
GMDS & -2.50769 & $1.41 \times 10^{-7}$ \\
SESN2 & -2.07768 & $1.54 \times 10^{-6}$ \\
VAV3 & -2.2753 & $5.05 \times 10^{-5}$ \\
LPIN1 & -2.46511 & $4.81 \times 10^{-6}$ \\
CYP2J2 & -3.04199 & $7.17 \times 10^{-7}$ \\
PIM1 & -2.38559 & $1.98 \times 10^{-9}$ \\
PAQR5 & -2.14799 & $1.366 \times 10^{-4}$ \\
RMND5B & -2.33016 & $5.89 \times 10^{-8}$ \\
NAPRT & -2.88074 & $1.33 \times 10^{-5}$ \\
SIM2 & -2.20672 & $8.21 \times 10^{-6}$ \\
CSTB & -4.16755 & $3.12 \times 10^{-10}$ \\
GPT2 & -2.13271 & $4.94 \times 10^{-6}$ \\
CES2 & -3.05714 & $7.74 \times 10^{-8}$ \\
YOD1 & -2.13921 & $9.91 \times 10^{-5}$ \\
TREX2 & -2.36119 & $2.738 \times 10^{-4}$ \\
\hline
\end{tabular}

Table IV. Differentially expressed genes interacting with fat mass and obesity-associated protein and negatively correlated with cancer susceptibility candidate 15 expression in esophagus cancer.

\begin{tabular}{llc}
\hline Gene names & R value & P-value \\
\hline GMDS & -0.46 & $7.39 \times 10^{-10}$ \\
CES2 & -0.453 & $1.46 \times 10^{-9}$ \\
NAPRT & -0.423 & $2.06 \times 10^{-8}$ \\
AGFG2 & -0.326 & $2.32 \times 10^{-5}$ \\
MXD1 & -0.317 & $4.03 \times 10^{-5}$ \\
LPIN1 & -0.31 & $5.86 \times 10^{-5}$ \\
SESN2 & -0.308 & $6.58 \times 10^{-5}$ \\
SIM2 & -0.302 & $9.17 \times 10^{-5}$ \\
CYP2J2 & -0.301 & $9.78 \times 10^{-5}$ \\
BARX2 & -0.284 & $2.51 \times 10^{-4}$ \\
RMND5B & -0.256 & $1.01 \times 10^{-3}$ \\
YOD1 & -0.251 & $1.27 \times 10^{-3}$ \\
\hline
\end{tabular}

which was initially inhibited by CASC15 overexpression in Eca-109 and KYSE450 cells (Fig. 4I-L).

CASC15 overexpression promotes ESCC cell proliferation and inhibits apoptosis via FTO.Functional analysis revealed that the ectopic expression of CASC15 enhanced cell proliferation and abated cell caspase-3 activity in Eca-109 and KYSE450 cells (Fig. 5A, B and E). Additionally, cell apoptotic rates were decreased by $\sim 0.5$-fold in Eca-109 and KYSE450 cells transfected with CASC15 overexpression plasmid compared with in
pcDNA-transfected cells (Fig. 5C and D). These data indicated that CASC15 exerted pro-proliferative and anti-apoptotic effects in ESCC cells. Furthermore, FTO-knockdown significantly weakened these CASC15-mediated pro-proliferative and anti-apoptotic effects (Fig. 5).

SIM2-knockdown reverses the effect of CASC15 silencing on ESCC cell proliferation and apoptosis. SIM2 expression was significantly decreased by $60 \%$ in ESCC tissues compared with in normal samples (Fig. 6A). Furthermore, SIM2 mRNA expression was negatively correlated with CASC15 expression in ESCC tissues ( $\mathrm{R}=-0.5183$; $\mathrm{P}=0.0003$; Fig. 6B). Additionally, SIM2 protein expression was markedly decreased in Eca109 and KYSE450 cells compared with in HET-1A cells (Fig. 6C). Additionally, knockdown efficiency analysis revealed that SIM2 protein expression was significantly decreased in Eca109 and KYSE450 cells transfected with si-SIM2 compared with in cells transfected with si-NC (Fig. 6D). To further investigate whether SIM2 was associated with the regulatory effects exerted by CASC15 on ESCC progression in vitro, Eca109 and KYSE450 cells were transfected with si-NC, si-CASC15\#1 or si-CASC15\#1 + si-SIM2. As presented in Fig. 6E, SIM2 protein expression was markedly increased in Eca109 and KYSE450 cells following CASC15-knockdown. The introduction of si-SIM2 markedly attenuated the stimulatory effect of CASC15-knockdown on SIM2 protein expression in Eca109 and KYSE450 cells (Fig. 6E). In addition, SIM2 downregulation partly reversed si-CASC15\#1-mediated anti-proliferative effects in Eca109 and KYSE450 cells (Fig. 6F). SIM2-knockdown led to a marked decrease in cell apoptotic rate from 24.57 to $14.83 \%$ in Eca109 cells transfected with si-CASC15\#1 (Fig. 6G and H). Additionally, a $~ 0.4$-fold (14.74 vs. 24.87\%) decrease in cell apoptotic rate was observed in KYSE450 cells co-transfected with si-CASC15\#1 and si-SIM2 compared with in cells co-transfected with si-CASC15\#1 and si-NC (Fig. 6G and H), suggesting that SIM2 silencing weakened the CASC15-knockdown-induced apoptosis in Eca109 and KYSE450 cells SIM2 interference also attenuated the CASC15-knockdown-induced caspase-3 activity promotion in Eca109 and KYSE450 cells (Fig. 6I). The results indicated that CASC15 suppression repressed ESCC progression in vitro by increasing SIM2.

\section{Discussion}

ESCC is the main subtype of esophageal cancer (31). lncRNAs have been implicated in the pathogenesis of ESCC and are involved in the regulation of ESCC progression (6). The current study aimed to elucidate a novel target for the treatment of ESCC. To the best of our knowledge, the present study was the first to demonstrate that CASC15-knockdown repressed ESCC progression, which was associated with the increase of SIM2 stability occurring through the decrease of FTO-mediated demethylation.

The results of the current study revealed that CASC15 expression was upregulated in ESCC tissue, which was consistent with the results of a previous study (13). Additionally, the present data demonstrated that high CASC15 expression was associated with poor survival outcomes in patients 
A

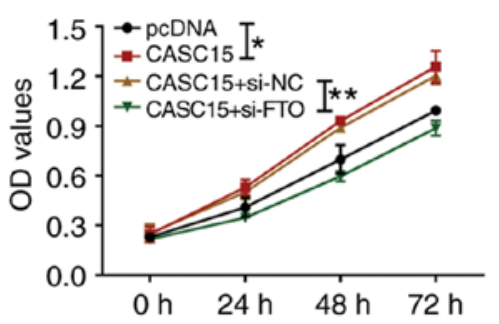

C

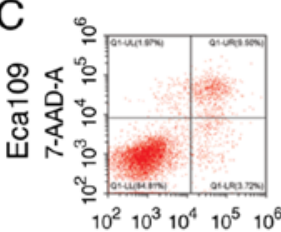

APC-A

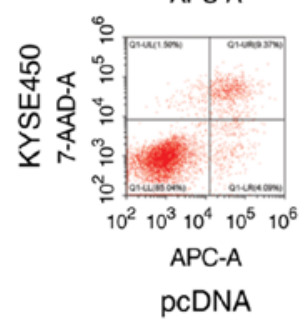

pcDNA
Eca109

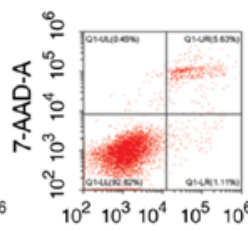

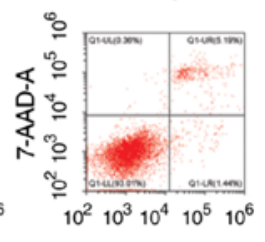

APC-A

CASC15

D

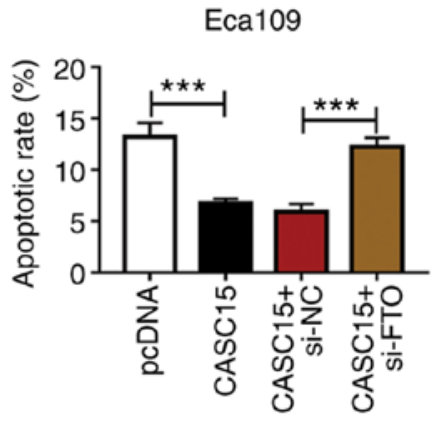

$\mathrm{E}$

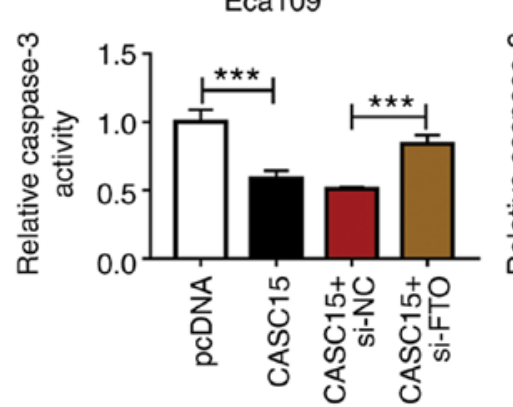

B

KYSE450
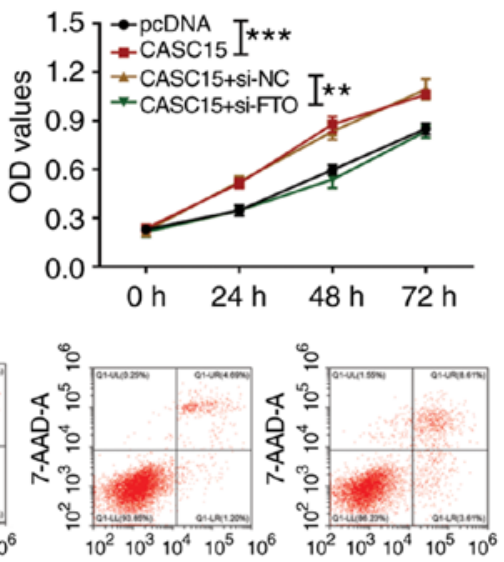

APC-A

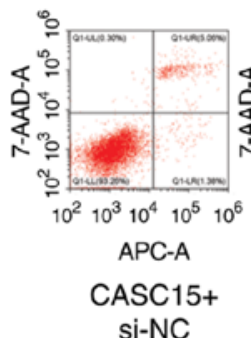

APC-A

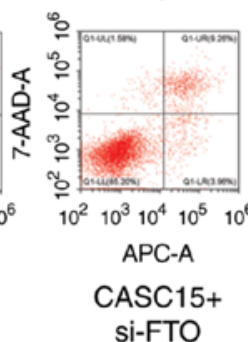

KYSE450

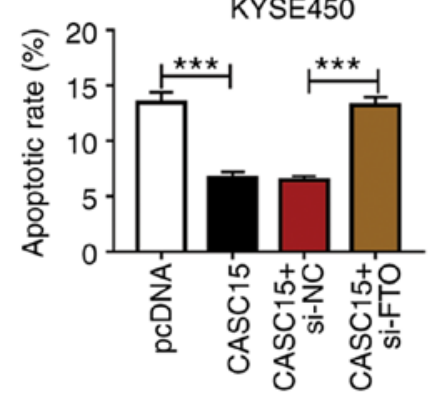

KYSE450

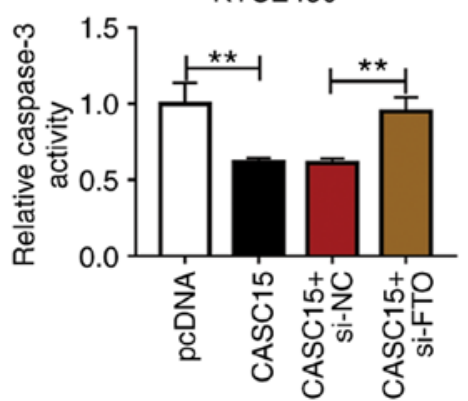

Figure 5. Effect of FTO-knockdown on CASC15-mediated esophageal squamous cell carcinoma cell proliferation and apoptosis. Eca109 and KYSE450 cells were transfected with pcDNA, CASC15, CASC15+si-NC and CASC15+si-FTO. Subsequently, cell viability, apoptosis and caspase-3 activity were detected via (A and B) MTT (data analyzed via two-way ANOVA followed by Bonferroni's post-hoc test), (C and D) flow cytometry and (E) caspase-3 assays, respectively. ${ }^{*} \mathrm{P}<0.05,{ }^{* *} \mathrm{P}<0.01$ and ${ }^{* * *} \mathrm{P}<0.001$. FTO, fat mass and obesity-associated; CASC15, cancer susceptibility candidate 15; si, small interfering RNA; NC, negative control; OD, optical density.

with ESCC, indicating that CASC15 had potential clinical diagnostic value in ESCC. Functional analyses revealed that CASC15-knockdown inhibited ESCC cell proliferation and promoted cell apoptosis in vitro. Xenograft tumor models are key tools for ESCC research (32). In vivo experiments of the present study demonstrated that CASC15 suppression hampered ESCC xenograft tumor growth. Consistently, previous studies have demonstrated that CASC15 exerted potential oncogenic effects in multiple types of cancer, including tongue squamous cell carcinoma, nasopharyngeal carcinoma, oral squamous cell carcinoma, bladder cancer, melanoma and cervical cancer $(9-12,33,34)$.

Previous studies have demonstrated that lncRNAs exert their functions by regulating mRNA biosynthesis, processing 
A

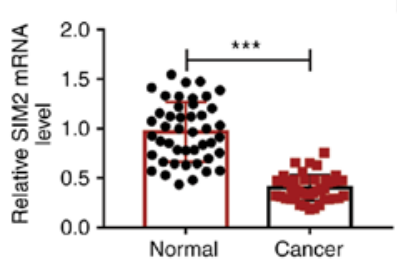

D

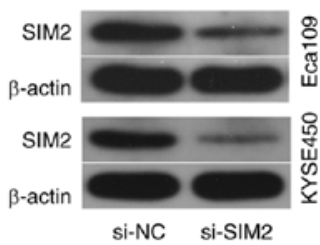

B

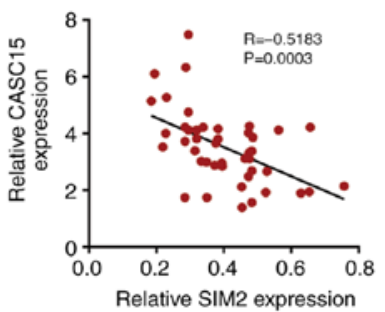

E

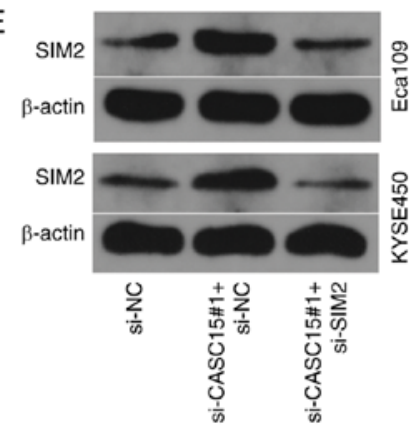

C

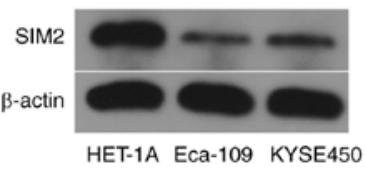

F
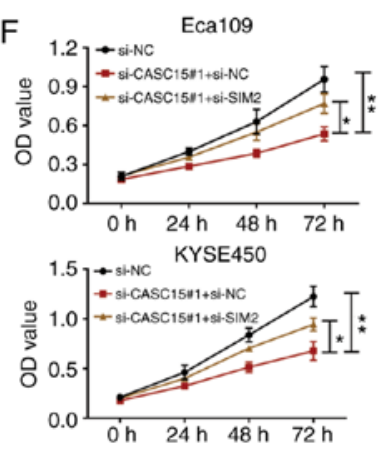
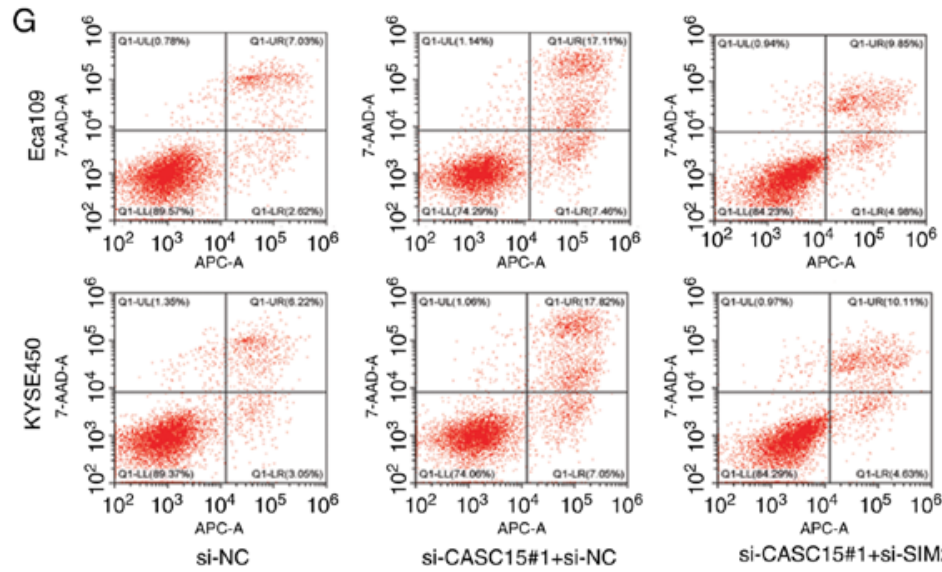

$\mathrm{H}$

Eca109
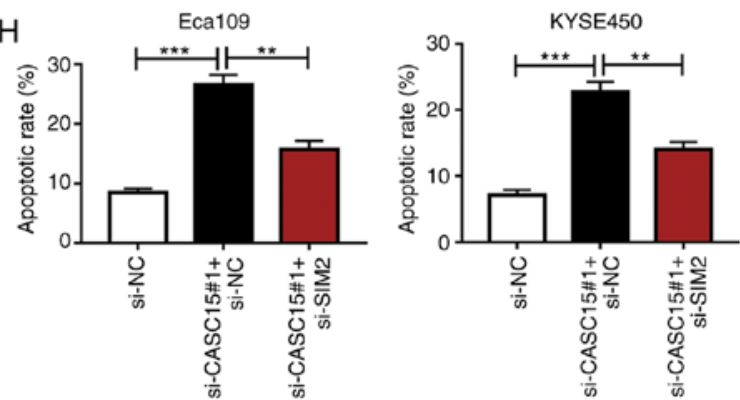

I

Eca109
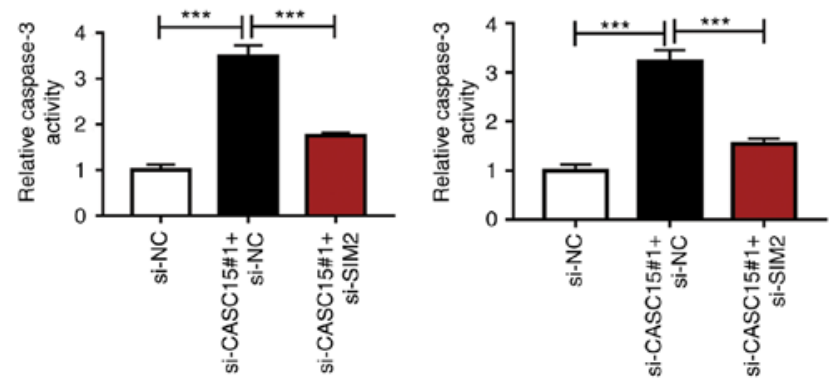

Figure 6. CASC15 depletion decreased the proliferation and promoted the apoptosis of ESCC cells by upregulating SIM2. (A) SIM2 expression was measured via reverse transcription-quantitative PCR in tumor and adjacent normal tissues isolated from patients with ESCC ( $\mathrm{n}=45$ ). (B) Linear correlation of CASC15 and SIM2 expression in ESCC tissues. (C) SIM2 protein expression was detected in HET-1A, Eca109 and KYSE450 cells via western blotting. (D) SIM2 protein expression was measured by western blotting at $48 \mathrm{~h}$ post transfection in Eca109 and KYSE450 cells transfected with si-NC or si-SIM2. Eca109 and KYSE450 cells were transfected with si-NC, si-CASC15\#1+si-NC or si-CASC15\#1+si-SIM2. (E) SIM2 protein abundance was examined via western blotting. (F) Cell proliferation was determined by performing an MTT assay in Eca109 and KYSE450 cells. Data analyzed via two-way ANOVA followed by Bonferroni's post-hoc test. (G and H) Apoptotic rate was detected via flow cytometry. (I) Relative caspase-3 activity was detected using the caspase-3 assay kit in Eca109 and KYSE450 cells. ${ }^{*} \mathrm{P}<0.05,{ }^{* *} \mathrm{P}<0.01$ and ${ }^{* * * *} \mathrm{P}<0.001$. CASC15, cancer susceptibility candidate 15; ESCC, esophageal squamous cell carcinoma; SIM2, single-minded 2; si, small interfering RNA; NC, negative control; OD, optical density. 
and stability in humans (35-37). N-m6A modification is also involved in regulating mRNA stability (38-40). Furthermore, FTO has been found to be a common demethylase targeting $\mathrm{N}$-methyladenosine $(41,42)$. FTO serves an important role in ESCC progression by mediating mRNA demethylation $(21,43)$. To the best of our knowledge, the current study was the first to confirm that CASC15 interacted with FTO to decrease SIM2 stability by FTO-mediated demethylation. FTO-knockdown markedly weakened CASC15-mediated pro-proliferative and anti-apoptotic effects in ESCC cells. Additionally, GEPIA database analysis revealed that SIM2 expression was decreased in ESCC tissues compared with in normal tissues. In line with the GEPIA data, Su et al (24) demonstrated that SIM2 expression was downregulated in ESCC. Furthermore, Tamaoki et al (23) revealed that downregulated SIM2 expression was associated with poor survival outcomes in patients with ESCC. In the present study, SIM2 downregulation weakened the detrimental effect of CASC15-knockdown on cell proliferation and inhibited the increase of apoptosis and caspase-3 activity induced by CASC15 depletion in ESCC cells, suggesting that SIM2 may exert antitumor effects in ESCC. The antitumor effects of SIM2 have also been demonstrated in cervical squamous cell carcinoma (44).

In conclusion, CASC15-knockdown decreased ESCC xenograft tumor growth in vivo. CASC15 promoted cell proliferation and inhibited apoptosis in ESCC by decreasing SIM2 stability via FTO-mediated demethylation. The current study provided a novel insight into the pathogenesis of ESCC and indicated a novel target for the treatment of ESCC. However, the present study only investigated the effect of CASC15 on ESCC tumorigenesis. In addition, the methylation status of CASC15 in the paired tumor and adjacent normal tissues in patients with ESCC was not analyzed in the present study. It is imperative to further examine the methylation status of CASC15 in paired tumor and adjacent normal tissues in patients with ESCC since FTO is a demethylase that can mediate m6A demethylation modification and elucidate the influence of CASC15 on ESCC tumor metastasis in vitro and in vivo. Additionally, whether CASC15 and FTO influence ESCC tumorigenesis and progression through the regulation of gene m6A demethylation requires further study.

\section{Acknowledgements}

Not applicable.

\section{Funding}

This study was supported by the National Natural Science Foundation of China (grant nos. 31801142 and 81900791), the Henan Key Project of Research and Development Plan (Science and Technology) (grant no. 192102310192) and the Henan Medical Science and Technology Joint Building Program (grant no. 2018020094).

\section{Availability of data and materials}

The datasets used and/or analyzed during the current study are available from the corresponding author on reasonable request.

\section{Authors' contributions}

BQ designed and performed most of the experiments, and wrote the manuscript. MD, ZW and JW contributed to the experimental work and data analysis. YX and DY made contributions to the study design, data analysis and manuscript revision. YJ performed some statistical analyses and assisted in acquiring patient information and sample collection. All authors have read and approved the final version to be published. Each author participated sufficiently in the work to take public responsibility for appropriate portions of the content.

\section{Ethics approval and consent to participate}

Written informed consent was obtained from all patients. The present study was approved by the Ethics Committee of the First Affiliated Hospital of Zhengzhou University (Zhengzhou, China) and conducted in accordance with the Declaration of Helsinki. The animal experiments were approved by the Institutional Animal Care and Use Committee of the First Affiliated Hospital of Zhengzhou University.

\section{Patient consent for publication}

Not applicable.

\section{Competing interests}

The authors declare that they have no competing interests.

\section{References}

1. Bray F, Ferlay J, Soerjomataram I, Siegel RL, Torre LA and Jemal A: Global cancer statistics 2018: GLOBOCAN estimates of incidence and mortality worldwide for 36 cancers in 185 countries. CA Cancer J Clin 68: 394-424, 2018.

2. Abnet CC, Arnold M and Wei WQ: Epidemiology of esophageal squamous cell carcinoma. Gastroenterology 154: 360-373, 2018.

3. Xie SH and Lagergren J: Risk factors for oesophageal cancer. Best Pract Res Clin Gastroenterol 36-37: 3-8, 2018.

4. Ohashi S, Miyamoto S, Kikuchi O, Goto T, Amanuma Y and Muto M: Recent advances from basic and clinical studies of esophageal squamous cell carcinoma. Gastroenterology 149: 1700-1715, 2015.

5. Feng Q, Zhang H, Yao D, Chen WD and Wang YD: Emerging role of non-coding RNAs in esophageal squamous cell carcinoma. Int J Mol Sci 30: 258, 2019.

6. Shen WJ, Zhang F, Zhao X and Xu J: LncRNAs and esophageal squamous cell carcinoma-implications for pathogenesis and drug development. J Cancer 7: 1258-1264, 2016.

7. Sun K and Zhang G: Long noncoding RNA CASC2 suppresses esophageal squamous cell carcinoma progression by increasing SOCS1 expression. Cell Biosci 9: 90, 2019.

8. Guan Z, Wang Y, Wang Y, Liu X, Wang Y, Zhang W, Chi X, Dong Y, Liu X, Shao S and Zhan Q: Long non-coding RNA LOC100133669 promotes cell proliferation in oesophageal squamous cell carcinoma. Cell Prolif 53: e12750, 2020.

9. Wu X, Ma J, Chen J and Huang H: LncRNA CACS15 regulates tongue squamous cell carcinoma cell behaviors and predicts survival. BMC Oral Health 19: 231, 2019.

10. Zhang X, Guo B, Zhu Y, Xu W, Ning S and Liu L: Up-regulation of plasma lncRNA CACS15 distinguished early-stage oral squamous cell carcinoma patient. Oral Dis 26: 1619-1624, 2019.

11. Sheng L and Wei R: Long Non-Coding RNA-CASC15 promotes cell proliferation, migration, and invasion by activating Wnt/ $\beta$-catenin signaling pathway in melanoma. Pathobiology 87 : 20-29, 2020. 
12. Zhang YN, Liu B, Jiang T and Li Q: Long non-coding RNA CASC15 promotes proliferation and induces apoptosis of cervical cancer cells through targeting miR-101-3p. Eur Rev Med Pharmacol Sci 24: 8627, 2020

13. Li Y, Shi X, Yang W, Lu Z, Wang P, Chen Z and He J: Transcriptome profiling of lncRNA and co-expression networks in esophageal squamous cell carcinoma by RNA sequencing. Tumour Biol 37: 13091-13100, 2016.

14. Chen XY, Zhang J and Zhu JS: The role of $\mathrm{m}^{6} \mathrm{~A}$ RNA methylation in human cancer. Mol Cancer 18: 103, 2019.

15. Dai D, Wang H, Zhu L, Jin H and Wang X: N6-methyladenosine links RNA metabolism to cancer progression. Cell Death Dis 9 : $124,2018$.

16. Li Z, Weng H, Su R, Weng X, Zuo Z, Li C, Huang H, Nachtergaele S, Dong L, Hu C, et al: FTO plays an oncogenic role in acute myeloid leukemia as a $\mathrm{N}^{6}$-methyladenosine RNA demethylase. Cancer Cell 31: 127-141, 2017.

17. Wang Q, Chen C, Ding Q, Zhao Y, Wang Z, Chen J, Jiang Z, Zhang Y, Xu G, Zhang J, et al: METTL3-mediated $\mathrm{m}^{6} \mathrm{~A}$ modification of HDGF mRNA promotes gastric cancer progression and has prognostic significance. Gut 69: 1193-1205, 2020.

18. Yoneda R, Ueda N, Uranishi K, Hirasaki M and Kurokawa R: Long noncoding RNA pncRNA-D reduces cyclin D1 gene expression and arrests cell cycle through RNA $\mathrm{m}^{6} \mathrm{~A}$ modification. J Biol Chem 295: 5626-5639, 2020.

19. Sun T, Wu Z, Wang X, Wang Y, Hu X, Qin W, Lu S, Xu D, Wu Y, Chen Q, et al: LNC942 promoting METTL14-mediated m(6)A methylation in breast cancer cell proliferation and progression. Oncogene 39: 5358-5372, 2020.

20. Zhu L, Zhu Y, Han S, Chen M, Song P, Dai D, Xu W, Jiang T, Feng L, Shin VY, et al: Impaired autophagic degradation of IncRNA ARHGAP5-AS1 promotes chemoresistance in gastric cancer. Cell Death Dis 10: 383, 2019.

21. Liu S, Huang M, Chen Z, Chen J, Chao Q, Yin X and Quan M: FTO promotes cell proliferation and migration in esophageal squamous cell carcinoma through up-regulation of MMP13. Exp Cell Res 389: 111894, 2020.

22. Takashima K, Fujii S, Komatsuzaki R, Komatsu M, Takahashi M, Kojima T, Daiko H, Minashi K, Chiwaki F, Muto M, et al: CD24 and CK 4 are upregulated by SIM2, and are predictive biomarkers for chemoradiotherapy and surgery in esophageal cancer. Int J Oncol 56: 835-847, 2020.

23. Tamaoki M, Komatsuzaki R, Komatsu M, Minashi K, Aoyagi K, Nishimura T, Chiwaki F, Hiroki T, Daiko H, Morishita K, et al: Multiple roles of single-minded 2 in esophageal squamous cell carcinoma and its clinical implications. Cancer Sci 109: 1121-1134, 2018.

24. Su P, Wen S, Zhang Y, Li Y, Xu Y, Zhu Y, Lv H, Zhang F, Wang $M$ and Tian Z: Identification of the key genes and pathways in esophageal carcinoma. Gastroenterol Res Pract 2016: 2968106, 2016.

25. Rice TW, Blackstone EH and Rusch VW: 7th edition of the AJCC Cancer Staging Manual: Esophagus and esophagogastric junction. Ann Surg Oncol 17: 1721-1724, 2010.

26. Rusch VW, Rice TW, Crowley J, Blackstone EH, Rami-Porta R and Goldstraw P: The seventh edition of the American Joint Committee on Cancer/International Union Against Cancer Staging Manuals: The new era of data-driven revisions. J Thorac Cardiovasc Surg 139: 819-821, 2010.

27. Chomczynski P and Sacchi N: The single-step method of RNA isolation by acid guanidinium thiocyanate-phenol-chloroform extraction: Twenty-something years on. Nat Protoc 1: 581-585, 2006
28. Livak KJ and Schmittgen TD: Analysis of relative gene expression data using real-time quantitative PCR and the 2(-Delta Delta C(T)) method. Methods 25: 402-408, 2001

29. Euhus DM, Hudd C, LaRegina MC and Johnson FE: Tumor measurement in the nude mouse. J Surg Oncol 31: 229-234, 1986.

30. Niu Y, Lin Z, Wan A, Chen H, Liang H, Sun L, Wang Y, Li X, Xiong XF, Wei B, et al: RNA N6-methyladenosine demethylase FTO promotes breast tumor progression through inhibiting BNIP3. Mol Cancer 18: 46, 2019.

31. Lagergren J, Smyth E, Cunningham D and Lagergren $P$ Oesophageal cancer. Lancet 390: 2383-2396, 2017

32. Lee NP, Chan CM, Tung LN, Wang HK and Law S: Tumor xenograft animal models for esophageal squamous cell carcinoma. J Biomed Sci 25: 66, 2018.

33. Xue MY and Cao HX: Long non-coding RNA CASC15 promotes nasopharyngeal carcinoma cell proliferation and metastasis by downregulating miR-101-3p. Eur Rev Med Pharmacol Sci 23: 8897-8904, 2019.

34. Yu X, Wang ZL, Han CL, Wang MW, Jin Y, Jin XB and Xia QH: LncRNA CASC15 functions as an oncogene by sponging miR-130b-3p in bladder cancer. Eur Rev Med Pharmacol Sci 23: 9814-9820, 2019.

35. Zhang X, Wang W, Zhu W, Dong J, Cheng Y, Yin Z and Shen F Mechanisms and functions of long non-coding RNAs at multiple regulatory levels. Int J Mol Sci 20: 5573, 2019.

36. Yao RW, Wang Y and Chen LL: Cellular functions of long noncoding RNAs. Nat Cell Biol 21: 542-551, 2019.

37. Akhade VS, Pal D and Kanduri C: Long noncoding RNA Genome organization and mechanism of action. Adv Exp Med Biol 1008: 47-74, 2017.

38. Chang G, Leu JS, Ma L, Xie K and Huang S: Methylation of RNA $\mathrm{N}^{6}$-methyladenosine in modulation of cytokine responses and tumorigenesis. Cytokine 118: 35-41, 2019.

39. Xiong X, Li X and Yi C: N(1)-methyladenosine methylome in messenger RNA and non-coding RNA. Curr Opin Chem Biol 45: 179-186, 2018.

40. Pan T: N6-methyl-adenosine modification in messenger and long non-coding RNA. Trends Biochem Sci 38: 204-209, 2013.

41. Jia G, Fu Y, Zhao X, Dai Q, Zheng G, Yang Y, Yi C, Lindahl T, Pan T, Yang YG and He C: N6-methyladenosine in nuclear RNA is a major substrate of the obesity-associated FTO. Nat Chem Biol 7: 885-887, 2011.

42. Fu Y, Jia G, Pang X, Wang RN, Wang X, Li CJ, Smemo S, Dai Q, Bailey KA, Nobrega MA, et al: FTO-mediated formation of N6-hydroxymethyladenosine and N6-formyladenosine in mammalian RNA. Nat Commun 4: 1798, 2013

43. Nagaki Y, Motoyama S, Yamaguchi T, Hoshizaki M, Sato Y, Sato T, Koizumi Y, Wakita A, Kawakita Y, Imai K, et al: $\mathrm{m}^{6}$ A demethylase ALKBH5 promotes proliferation of esophageal squamous cell carcinoma associated with poor prognosis. Genes Cells 25: 547-561, 2020

44. Nakamura K, Komatsu M, Chiwaki F, Takeda T, Kobayashi Y, Banno K, Aoki D, Yoshida T and Sasaki H: SIM2l attenuates resistance to hypoxia and tumor growth by transcriptional suppression of HIF1A in uterine cervical squamous cell carcinoma. Sci Rep 7: 14574, 2017.

This work is licensed under a Creative Commons Attribution-NonCommercial-NoDerivatives 4.0 International (CC BY-NC-ND 4.0) License. 